\title{
PARTICIPACIÓN EN ORGANIZACIONES SOCIALES EN CHILE. ¿UNA ALTERNATIVA PARA MEJORAR EL BIENESTAR ECONÓMICO DE LOS HOGARES? ${ }^{1}$ IS PARTICIPATION IN SOCIAL ORGANIZATIONS AN ALTERNATIVE
TO IMPROVE HOUSEHOLD ECONOMIC WELFARE IN CHILE?
}

CÉSAR A. SALAZAR*3

MónICA M. JAIME**

\begin{abstract}
Resumen
El propósito de este trabajo es conocer los determinantes de la participación en organizaciones sociales en Chile, y evaluar si la participación ha conducido a una mejora en el bienestar económico de los hogares utilizando el enfoque del capital social. Se estimaron modelos de participación e ingreso en el caso de las organizaciones económicas y vecinales y un modelo de ecuaciones simultáneas en el caso de las organizaciones recreativo-culturales, debido a la existencia de endogeneidad del ingreso en la ecuación de participación. El modelo simultáneo propuesto incluye estimadores Two Stage Probit Least Squares, adecuados para corregir los sesgos derivados de la simultaneidad cuando una variable dependiente es dicotómica y la otra continua. Los resultados indican que la edad, educación, tenencia de una vivienda propia, zona de residencia y la percepción con respecto a la seguridad y convivencia entre los vecinos tienen una fuerte incidencia en la probabilidad de participar, y que existe una relación positiva entre ingreso y asociatividad económica.
\end{abstract}

Palabras clave: Capital social, organizaciones de la sociedad civil, efectos en el bienestar.

* Departamento de Economía y Finanzas, Facultad de Ciencias Empresariales, Universidad del Bío-Bío. Avenida Collao No 1202, Casilla 5-C, Concepción (Chile). Teléfono: (56) (41)2731272, Fax: (56) (41)2731087. E-mail: csalazar@ubiobio.cl

** Escuela de Administración y Negocios. Universidad de Concepción, Campus Chillán. Avenida Vicente Méndez 595, casilla 537, Chillán (Chile). Teléfono: (56) (42) 208733, Fax: (56) (42)275305. E-mail: mjaime@udec.cl

1 Agradecemos los valiosos comentarios realizados por dos árbitros evaluadores anónimos.

2 Esta investigación ha sido financiada por la Dirección de Investigación de la Universidad del Bío-Bío, Proyecto DIUBB 081217 1/I.

3 En esta investigación se utilizó información de la Encuesta Panel de Caracterización Socioeconómica Nacional. Los autores agradecen al Ministerio de Planificación, propietario intelectual de la Encuesta, haberles permitido disponer de la base de datos. Todos los resultados del estudio son de responsabilidad de los autores, y en nada comprometen a dicho Ministerio. 


\begin{abstract}
This research aims to find the determinants of participation on social organizations in Chile through a social capital approach, as well as to evaluate the existence of a positive effect between participation and household welfare. In the case of economic and local organizations several regressions for participation and income were run. Due to the existence of endogenity of income in participation equation, it was estimated a simultaneous model for recreational organizations. The proposed model includes Two Stage Probit Least Squares estimates suitable to correct for simultaneity bias when one of the variables is continuous and the other is dichotomous. Econometric results point out that age, schooling, house ownership, place of residence and individual perception of both social intercourse and safety in their neighborhoods have a meaningful effect on participation. The study also reported on the positive effect between participation in economic organizations and household welfare.
\end{abstract}

Key words: Social capital, social organizations, welfare effects.

JEL Classification: D6, N3, O1, Z0.

\title{
I. INTRODUCCIÓN
}

El Programa de las Naciones Unidas para el Desarrollo define la asociatividad como toda organización voluntaria y no remunerada de personas o grupos de ellas que establecen un vínculo explícito con el fin de conseguir un objetivo común. A nivel mundial, se han realizado diversos estudios para registrar y clasificar las organizaciones en función de sus objetivos, y se ha reconocido ampliamente el aporte que éstas realizan en la creación de riqueza y de empleo, además de su contribución a la cohesión social de un país (PNUD, 2000; Portes y Landolt, 2000). No obstante, han sido escasos los esfuerzos dirigidos a analizar el proceso inherente a la formación de las mismas desde una perspectiva económica y a evaluar el impacto que éstas podrían tener en el desarrollo económico y social en el caso chileno.

La asociatividad como instrumento para resolver de manera descentralizada algunos dilemas sociales no es una propuesta reciente. Los diferentes tipos de organizaciones que han tenido lugar a lo largo del tiempo no han sido un resultado del accionar de los gobiernos, ni del comportamiento de los mercados; antes bien, han sido consolidadas a través del voluntarismo, aspecto que adquiere un soporte teórico en la Tragedia de los Bienes Comunes (Hardin, 1968), la Lógica de la Acción Colectiva (Olson, 1965) y en el Dilema del Prisionero, enfoques que describen situaciones en las que todo el mundo podría mejorar en un escenario de cooperación.

En los últimos años, a raíz de una serie de reformas que tenían como objetivo acercar a las personas a las organizaciones, los gobiernos han visto la importancia de retomar este enfoque e integrar a la sociedad civil en la gestión de las políticas públicas, aspecto que hace necesario el fortalecimiento de las organizaciones y el establecimiento de políticas para fomentar el voluntariado. 
Las relaciones de participación, asociatividad y confianza que tienen lugar en una sociedad hacen referencia a sus niveles de capital social, un activo que junto al capital humano, capital natural, capital físico y capital financiero pueden explicar los niveles de desarrollo económico y social de un país (Aker, 2007; Knack y Keefer, 1997). Si bien existen diversas visiones con respecto a este concepto debido al potencial que presenta para generar externalidades en diferentes ámbitos, Putnam (1995) recoge la mayor parte de ellas, al definir el capital social como "las características de las organizaciones sociales tales como interrelaciones, normas y confianza que facilitan la cooperación y coordinación para beneficio mutuo".

El propósito de este trabajo es conocer los determinantes de la participación en organizaciones sociales en Chile, y evaluar si la participación ha conducido a una mejora en el bienestar económico utilizando el enfoque del capital social. Para tal efecto, a partir de la información suministrada por la encuesta Panel CASEN 2006, se estimarán econométricamente los determinantes de la participación y del ingreso de un individuo, a través de un modelo de ecuaciones simultáneas que corrige los sesgos derivados de la doble causalidad que podría existir entre el ingreso y la participación.

Los resultados obtenidos proveerán información de relevancia para el fortalecimiento de las organizaciones existentes, la promoción de nuevas instancias de participación, y para la incorporación de la sociedad civil en el diseño y gestión de las políticas públicas. Este artículo se encuentra estructurado de la siguiente manera: en la sección dos se presenta el enfoque teórico de la participación como medida de capital social. La sección tres presenta el modelo económico de la participación y el bienestar material. Los detalles con respecto a la aplicación econométrica son presentados en la sección cuatro. En la sección cinco se exponen y discuten los resultados. Finalmente, se presentan las conclusiones del estudio.

\section{Capital Social y Participación}

En la literatura del capital social se ha destacado su contribución a la protección y manejo de los recursos, a la capacidad de generar acuerdos en las diferentes sociedades y a la promoción de actitudes de respeto y confianza social, lo que constituye una manera de dinamizar y potenciar la vida social de un país. De acuerdo con Woolcock (1998), una sociedad dotada con altos niveles de capital social será más segura, exhibirá mejores índices de calidad de vida, estará mejor gobernada y sus habitantes alcanzarán mayores niveles de bienestar. De la misma manera, Kliksberg (1999) señala que el capital social incentiva en las personas actitudes positivas como el cuidado de los espacios públicos, el pago de los impuestos, así como la presencia de altos niveles de asociatividad, y es precisamente este comportamiento cooperativo el que puede producir un aumento en el bienestar general de un país.

Knack y Keefer (1997) encuentran que tanto la confianza como las normas de cooperación cívica han tenido un impacto positivo en el crecimiento económico en un amplio grupo de países, y que estos componentes del capital social son mayores en sociedades que exhiben menores diferencias en términos étnicos y de ingreso. Por su parte, Bebbington (2005) analiza la importancia del capital 
social en la generación de vínculos que trascienden las estructuras sociales convencionales, generando las condiciones de movilidad social que son necesarias para la superación de la pobreza.

En la mayoría de los estudios empíricos que tienen como objetivo la medición del capital social se utiliza como variable proxy la "confianza interpersonal" como una medida de stock y la "participación en organizaciones sociales" como una medida de flujo (Lederman, 2005). Si bien ambas variables constituyen una buena representación del capital social, en realidad conforman dos enfoques distintos que han sido objeto de debate durante las últimas décadas.

De acuerdo con el primer enfoque, la confianza interpersonal constituye un capital debido a que proporciona mayores beneficios a aquellos individuos que establecen este tipo de relaciones, y se caracteriza por tener un efecto multiplicador ya que puede ser acumulada a lo largo del tiempo. Gordon (2005) señala que la confianza es el sustento de las relaciones de reciprocidad en que se basa el capital social, y que la norma de reciprocidad basada en la confianza ayuda a resolver problemas relacionados con los bienes públicos en una comunidad. Desde este punto de vista, las organizaciones voluntarias constituyen instrumentos para la formación de capital social, al disminuir la incertidumbre con respecto a la conducta de los otros y dar lugar a un proceso de familiaridad y cercanía, en el cual los individuos empiezan a compartir sus identidades en torno a un objetivo común.

Por su parte, el enfoque de la participación señala que ésta es importante para aquellos grupos que tienen una menor oportunidad de influir en las decisiones de políticas públicas, al generar formas de identidad compartida y fomentar la capacidad de emprender una acción colectiva (PNUD, 2000). La pertenencia a grupos genera interacciones entre individuos facilitando la transmisión del conocimiento, aumentando los niveles de capital humano y el desarrollo de la confianza, la cual mejora el funcionamiento de los mercados (Alesina y La Ferrara, 2000a). La unión a una red social constituye una de las expresiones más comunes de inversión en capital social, ya que disminuye la distancia social, creando confianza, lealtad, altruismo y cooperación (Glaeser et al., 2002). Desde esta perspectiva, es posible afirmar que la asociatividad per se, al contribuir al fortalecimiento de las capacidades sociales de un país, constituye una proxy del capital social, de acuerdo a la definición de Putnam (1995).

La participación como medida de capital social puede ser vista como una estrategia de devolución de la autoridad del poder, recursos y de distribución de derechos desde niveles locales de gobierno a la sociedad civil (Kothari, 2001). De acuerdo con Khanal (2005), las actividades de participación pueden incluir situaciones donde un individuo toma parte de una acción conjunta, comparte algo en común, o se involucra activamente con otros miembros de su comunidad en torno a decisiones que afectan a todos.

Con respecto al ámbito de acción, el PNUD (2000) ha realizado una clasificación de las organizaciones sociales. Entre ellas cabe destacar las organizaciones con fines económicos, en las que los individuos persiguen mejoras en sus condiciones laborales, productivas, tecnológicas, capacidad de negociación y representación política. En segundo lugar, las asociaciones vecinales, en las que los individuos buscan un mejoramiento en las condiciones físicas (acceso a servicios básicos y equipamiento comunitario) y del medio ambiente social (seguridad y relación con los vecinos) de sus comunidades de residencia. En tercer lugar, la asociatividad 
recreativo-cultural, que busca la creación de instancias para el uso del tiempo libre y el desarrollo de formas de expresión artística y cultural.

Finalmente, la participación hace posible que los individuos accedan a una serie de recursos de tipo social, colectivo, económico y cultural, pero impone a su vez una serie de costos que van desde las cuotas de membresía hasta la renuncia al tiempo disponible para la generación de ingreso y otras actividades (Yusuf, 2008). Debido a la diversidad de fines al interior de los distintos tipos de organizaciones, es de esperarse que la participación produzca efectos diferenciados en términos de costos y beneficios. Un análisis de los costos y beneficios de participar es presentado en Behera (2005) y Behera y Engel (2006).

\section{Modelación Económica de la Participación y el Bienestar ECONóMico}

\subsection{Participación y capital social}

En esta aplicación, la decisión de participar en una organización será modelada a través del enfoque de la función índice. De acuerdo con este enfoque, los individuos realizan una comparación entre los costos y beneficios asociados a la participación. Se asume que el beneficio neto de participar en una organización se puede modelar como una variable latente:

$$
y_{1 i}^{*}=\gamma_{1} y_{2 i}+\beta_{1}^{\prime} X_{1}+\varepsilon_{1}
$$

donde: $y_{1 i}^{*}$ hace referencia al beneficio neto de participar, el cual no es observable pero se puede modelar como una variable dicotómica $y_{1 i}$, que toma el valor de 1 si el individuo $i$ participa en una organización $\left(y_{1 i}^{*}>0\right)$, y toma el valor de 0 si no participa en una organización $\left(y_{1 i}^{*} \leq 0\right) ; y_{2 i}$ es una variable continua que refleja el bienestar económico del individuo $i ; X_{1}$ es una matriz de variables exógenas que contiene un conjunto de características individuales, familiares y de la comunidad de residencia del individuo $i ; \gamma_{1}$ es el parámetro asociado a la variable bienestar económico; $\beta_{1}^{\prime}$ es un vector de parámetros asociados al conjunto de variables exógenas. Finalmente, $\varepsilon_{1}$ corresponde al término error. Esta expresión puede reescribirse de la siguiente manera:

$$
\begin{aligned}
& \mathrm{P}[\text { participar }]=\mathrm{P}\left[y_{1 i}=1\right]=\mathrm{P}\left[y_{1 i}^{*}>0\right]=\mathrm{P}\left[\varepsilon_{1}>-\left(\gamma_{1} y_{2 i}+\beta_{1}^{\prime} X_{1}\right)\right] \\
& \mathrm{P}[\text { participar }]=\mathrm{P}\left[y_{1 i}=1\right]=\mathrm{P}\left[\varepsilon_{1}<\gamma_{1} y_{2 i}+\beta_{1}^{\prime} X_{1}\right]=F\left(\gamma_{1} y_{2 i}+\beta_{1}^{\prime} X_{1}\right)
\end{aligned}
$$

donde: $\mathrm{P}[\cdot]$ es la probabilidad de que un individuo decida participar en una organización, y $F$ es la función de distribución acumulada de $\varepsilon_{1}$.

La problemática de la participación ha sido abordada en una gran cantidad de aplicaciones empíricas. Con respecto a sus determinantes, Glaeser et al. (2002) estiman un modelo de inversión individual para analizar el proceso de formación de capital social, encontrando que variables como la edad, el grado de 
sociabilidad de la ocupación y la movilidad afectan los niveles de capital social. Weinberger y Jütting (1999) analizan los determinantes de la participación en grupos de desarrollo local, siendo ésta explicada principalmente por grupos de ingreso medio y por la existencia de redes sociales al interior de las localidades. Resultados similares se pueden ser observados en Jütting (2003).

Khanal (2005) analiza los factores que influencian los niveles de participación en actividades de administración forestal comunitaria en Nepal a partir de un conjunto de variables de carácter biofísico, económico y social. Del mismo modo, Katungi et al. (2007) identifican los factores que influencian la participación de los hogares en organizaciones locales, la intensidad de participación, y la intensidad de las redes sociales de los hogares en Uganda, para concluir que las características de los hogares y la homogeneidad de las comunidades influencian las distintas dimensiones del capital social. (Mayor información con respecto a los niveles de participación puede ser encontrada en Davis y Negash, 2007; Alesina y La Ferrara, 2000b).

Lederman (2005) investiga los determinantes de la participación de la población argentina en organizaciones sociales, así como los factores que explican la disposición a confiar entre sus miembros, haciendo énfasis en el rol que cumplen el ingreso y la riqueza. Por su parte, Agarwal (2001) estudia el desempeño de las organizaciones, específicamente el proceso de inclusión de los grupos más desfavorecidos, evaluando el impacto que tiene sobre la eficiencia la existencia de fenómenos como la desigualdad de género, de raza e ingreso. Finalmente, Haddad y Maluccio (2003) estudian la importancia que tiene la confianza en la decisión de afiliarse a un grupo, la capacidad de los grupos para generar confianza y el efecto de la afiliación y la confianza en los grupos sobre el bienestar material de las familias. (Para mayor información con respecto al papel de la confianza, ver: Alesina y La Ferrara, 2000a; Cárdenas 2003).

\subsection{Bienestar económico y capital social}

Se tiene evidencia de que los elementos que conforman el capital social pueden potencialmente ayudar a mejorar los resultados económicos a través de tres mecanismos: en primer lugar, se observa que las comunidades con vínculos más fuertes entre sus miembros se encuentran mejor preparadas para entablar una acción cooperativa, por lo que se tiene una menor probabilidad de que se produzcan situaciones como la "tragedia de los comunes" o comportamientos "free rider" (Grootaert, 1997; Woolcock, 1998; Aker, 2007).

En segundo lugar, una actividad asociativa más fuerte puede reducir los problemas de información imperfecta, disminuyendo los costos económicos y sociales asociados a transacciones de insumos, créditos, tierra y nueva tecnología (Aker, 2007; Narayan, 1997; Narayan y Pritchett, 1999; Grootaert, 1999). Finalmente, en comunidades con relaciones de confianza y vínculos más fuertes es más probable compartir riesgos y desarrollar medidas informales de protección frente a siniestros, con los cuales las familias podrían alcanzar retornos esperados más altos y mitigar los efectos negativos que se tienen como consecuencia de shocks exógenos (Townsend, 1994).

El marco analítico propuesto para estudiar la relación entre las medidas de capital social y el bienestar económico está basado en los trabajos de Narayan 
y Pritchett (1999) y Grootaert et al. (2002), quienes derivan su análisis a partir del problema de la maximización de la utilidad familiar. De acuerdo con este enfoque, el capital social actúa como una clase de activo disponible para que la familia pueda generar ingresos. Se asume que un hogar se encuentra dotado de capital físico, capital humano y capital social; de este modo, la relación entre bienestar económico y capital social puede ser representada a través de la siguiente ecuación:

$$
y_{2 i}=\gamma_{2} y_{1 i}^{*}+\beta_{2}^{\prime} X_{2}+\varepsilon_{2}
$$

donde: $y_{2 i}$ corresponde al nivel de bienestar económico asociado al individuo $i ; y_{1 i}^{*}$ es la dotación de capital social, medido a través de la participación en organizaciones; $\gamma_{2}$ es el parámetro de la variable asociada a la participación; $\mathrm{X}_{2}$ corresponde a una matriz de variables exógenas relacionadas a características individuales, familiares y de nivel de comunidad, así como la dotación de otros activos; $\beta_{2}^{\prime}$ es un vector de parámetros de variables exógenas; y $\varepsilon_{2}$ corresponde al término error.

Se asume también que los precios de los insumos y servicios se encuentran dados en el modelo, pero que el acceso a ellos puede ser influenciado por los niveles de capital social y otras características de la familia; de esta manera, las redes construidas a través de interacciones generan beneficios medibles para los participantes a nivel individual, generando directa o indirectamente mayores niveles de bienestar.

La literatura ha demostrado que las diferencias en el bienestar económico no pueden ser completamente explicadas a través de los enfoques tradicionales de capital humano, físico, financiero y natural, por lo que existe un interés creciente en estudiar la forma en que el capital social puede contribuir a explicar los resultados económicos de los agentes.

Grootaert y Narayan (2004) estudian empíricamente el impacto del capital social en el bienestar familiar en Bolivia, encontrando evidencia de que el capital social incrementa el gasto familiar per cápita y reduce la pobreza, y que este efecto es mayor en el caso de las familias más pobres. Por su parte, Aker (2007) observa una relación positiva entre el gasto familiar per cápita y las medidas de capital social a nivel familiar, y una relación negativa entre la probabilidad de ser pobre y las distintas medidas de capital social en Tanzania.

Con respecto a los efectos diferenciados de los grupos en el bienestar familiar, Amudavi (2007) encuentra que la participación en grupos formales conlleva mayores niveles de bienestar con respecto a organizaciones de carácter informal; sin embargo, el estudio no permite concluir nada con respecto al efecto de la participación en grupos locales. Degli (2007) utiliza variables de capital social cuantitativas y cualitativas para medir el efecto que éstas generan en las medidas de bienestar material objetivas y subjetivas para una muestra de familias italianas, concluyendo que ambas medidas de capital social son efectivas para incrementar el bienestar económico de los hogares.

$\mathrm{Hu}$ y Jones (2004) investigan la relación existente entre capital social y bienestar económico en Uganda utilizando medidas de capital social aditivas y 
multiplicativas ${ }^{4}$, para concluir que si bien el índice multiplicativo es significativo, la afiliación a organizaciones solo es significativa cuando esta es incorporada como medida de capital social aditiva. Finalmente, utilizando este mismo tipo de mediciones, Yusuf (2008) verifica la existencia de una relación positiva y significativa de la afiliación en organizaciones sobre el gasto familiar per cápita como medida de bienestar en Nigeria, lo que permite concluir que el capital social tiene un efecto positivo sobre el bienestar, y es un factor de gran importancia para mejorar el estándar de vida de los hogares.

\subsection{Problema de simultaneidad entre el capital social y el bienestar económico}

Es probable que exista una doble causalidad entre las medidas de capital social y bienestar económico. En primer lugar, el capital social presenta características de un bien de consumo que demanda tiempo y dinero, por lo que es posible que su demanda se incremente con el ingreso. En segundo lugar, los individuos que participan tienen una mayor oportunidad de aumentar sus ingresos con respecto a los que no participan. Desde esta perspectiva, los resultados obtenidos al emplear técnicas de estimación estándar en presencia de simultaneidad serán sesgados e inconsistentes (Greene, 1999).

Este problema puede ser corregido al utilizar métodos de estimación de ecuaciones simultáneas, que en la mayoría de los casos incorporan variables endógenas que son observadas y continuas. Sin embargo, dada la naturaleza del problema, es posible observar que mientras una de las variables es continua (bienestar), la otra es de carácter discreto (participación). Maddala (1983) propone un enfoque teórico que hace posible corregir este sesgo de simultaneidad a partir de la estimación de un modelo Probit para la variable dependiente cualitativa y el método Mínimos Cuadrados Ordinarios (MCO) para la variable endógena continua. Este procedimiento de estimación es denominado Two Stage Probit Least Squares (Keshk, 2003). El sistema de ecuaciones a estimar es definido a partir de las ecuaciones (1) y (4).

$$
\begin{aligned}
& y_{1 i}^{*}=\gamma_{1} y_{2 i}+\beta_{1}^{\prime} X_{1}+\varepsilon_{1} \\
& y_{2 i}=\gamma_{2} y_{1 i}^{*}+\beta_{2}^{\prime} X_{2}+\varepsilon_{2}
\end{aligned}
$$

donde: $y_{1 i}^{*}$ y $y_{2 i}$ corresponden a las variables endógenas del sistema. Debido a que $y_{1 i}^{*}$ es observado solamente como una variable dicotómica, y considerando $\sigma_{1}^{2}$ como la varianza de $\varepsilon_{1}$, las ecuaciones estructurales se pueden reescribir de la siguiente manera:

4 Las variables multiplicativas se construyen considerando información de un conjunto de variables orientadas a medir un objetivo común. A partir de esta información se construye un índice multiplicativo que trata de representar la variación conjunta de todas estas variables. Cuando las variables se incorporan aditivamente, son consideradas separadamente en el proceso de estimación. 


$$
\begin{gathered}
y_{1 i}^{* *}=\frac{\gamma_{1}}{\sigma_{1}} y_{2 i}+\frac{\beta_{1}^{\prime}}{\sigma_{1}} X_{1}+\frac{\varepsilon_{1}}{\sigma_{1}} \\
y_{2 i}=\gamma_{2} \sigma_{1} y_{1 i}^{* *}+\beta_{2}^{\prime} X_{2}+\varepsilon_{2}
\end{gathered}
$$

Finalmente, se efectúa un proceso de corrección de errores, derivando la matriz de covarianzas asintótica siguiendo el procedimiento sugerido en Amemiya (1979). Un resumen detallado de este procedimiento se encuentra disponible en el apéndice A.

\section{Aplicación}

Para llevar a cabo las estimaciones econométricas se utilizó la Encuesta Panel de Caracterización Socioeconómica Nacional (Panel CASEN 2006) ${ }^{5}$. Con base en lo anterior, el nivel de capital social de un hogar fue medido a través de la participación en organizaciones en las que el jefe de hogar declara pertenecer. Considerando la información registrada en esta encuesta, se tiene una muestra de 3.766 hogares, de los cuales el 36,38\% declaró participar por lo menos en una organización.

Con respecto a los determinantes de la participación, se estimaron modelos para el total de organizaciones 6 y para algunas categorías de organización propuesta por el PNUD (2000). La ecuación a estimar es de la forma ${ }^{7}$ :

$$
\begin{aligned}
& \mathrm{P}\left(\text { Part }_{i}=1\right)=f\left(E D A D_{i}, E D A D_{i}^{2}, E S C_{i}, E S C_{i}^{2}, H O M_{i}, S E G_{i}, V E C_{i}, S A L U D_{i},\right. \\
& \text { APOYO }_{i}, \text { NEST }_{i}, \mathrm{HOG}_{i}, \mathrm{HOG}_{i}, \mathrm{HOG}_{i}, \mathrm{HOG}_{i}, \mathrm{HOG}_{i}, \mathrm{DESC}_{i}, \mathrm{CASA}_{i} \text {, } \\
& \left.L N(I N G)_{i}, R U R A L_{j}, D E S_{j}, D E S I G_{j}, R E G 3, R E G 7, R E G 8\right) \text {, }
\end{aligned}
$$

5 En esta encuesta se formula la siguiente pregunta: "La siguiente es una lista de organizaciones que cuentan con personalidad jurídica, estatutos, eligen directiva, etc. ¿A cuáles de estas organizaciones pertenece usted? (la pertenencia definida como estar inscrito en la organización)".

6 La totalidad de las organizaciones consultadas son las siguientes: Organización de vecinos de un barrio o comuna; Centros de Madres; Grupo de Mujeres; Centros de alumnos o Federación de Estudiantes; Club Deportivo y recreativo; Agrupación cultural o relacionada con la creación artística; Grupo de juegos, hobby o pasatiempos; Partido político; Grupo Religioso o de Iglesia; Organización de Voluntariado; Colegio Profesional; Agrupación de empresarios; Grupo de Salud o autoayuda; Organizaciones de Adulto Mayor; Organizaciones de Personas con Discapacidad; Centro de Padres y Apoderados; Organización campesina, grupos de adelantos rurales; ONG, Fundación o Corporación; Agrupación o asociación indígena; Sindicatos; Cooperativa, taller productivo, laboral o microempresa; Organizaciones ambientalistas, juntas de vecinos y otras.

7 En la definición del set de regresores final se hicieron estimaciones incluyendo variables explicativas adicionales como: estado civil del jefe de hogar, disponibilidad de televisión por cable en el hogar, población en número de habitantes en la provincia, así como un conjunto de categorías ocupacionales. Los resultados no varían al excluir estas variables, por lo que fueron eliminadas del modelo final. 
donde: $P\left(\right.$ Part $\left._{i}=1\right)$ es la probabilidad de que el individuo $i$ participe en una organización; Part $_{i}$ es una variable binaria que toma el valor de 1 si el individuo $i$ pertenece al menos a una organización, y 0 si no pertenece a ninguna ${ }^{8}$.

Con respecto al vector de variables individuales, se definen $E D A D_{i}$ y $E D A D_{i}^{2}$ (al cuadrado) como el número de años de vida del jefe de hogar; esta especificación tiene como propósito definir una relación no lineal entre capital social y edad. Para capturar el efecto del nivel de educación sobre la decisión de afiliación, se especificaron las variables $E S C_{i}$ y $E S C_{i}^{2}$ (al cuadrado), las cuales hacen referencia a los años de escolaridad formal del jefe de hogar. Al respecto, individuos con mayor edad y capital humano podrían obtener un mayor retorno desde las interacciones sociales ya que la expresión de opiniones y argumentos más convincentes se fortalecen con la experiencia y educación (Weinberger y Jütting, 1999; Behera y Engel, 2006; Glaeser et al., 2002).

Para controlar por el género del jefe de hogar, se define la variable dicotómica $\mathrm{HOM}_{i}$ tomando el valor 1 si el jefe de hogar es hombre, y 0 si es mujer.

Teniendo en consideración que la participación puede ser afectada por la confianza (Glaeser et al., 2002; Lederman, 2005), y que la confianza puede ser utilizada para explicar otros resultados económicos, incluyendo la formación de grupos (Haddad y Maluccio, 2003), se incluye un conjunto de variables que reflejan la propensión a confiar de los jefes de hogar. De esta manera, se definen las variables categóricas $S E G_{i}$ (seguridad en el barrio) y $V E C_{i}$ (relación con los vecinos), las cuales toman el valor de 3 cuando la persona declara que la situación ha mejorado mucho, el valor de 2 cuando la situación ha mejorado, 1 cuando se percibe que la situación sigue igual y 0 cuando se afirma que ha empeorado.

En la misma dirección se define la variable dicotómica $S A L U D_{i}$, que toma el valor de 1 si la persona se vio enfrentada durante los últimos cinco años a algún problema de salud grave, y el valor de 0 en otro caso. La ocurrencia de un trauma en el estado de salud podría afectar el nivel de confianza del individuo y de esta manera influenciar su capacidad para entablar relaciones sociales (Alesina y La Ferrara, 2000a). Finalmente se define $A P O Y O_{i}$, que toma el valor de 1 si el individuo declara acudir a personas diferentes a su familia en caso de enfrentar un problema de salud que demande grandes cuidados y recursos, y el valor de 0 en otro caso. De acuerdo a Lederman (2005), un individuo posee confianza interpersonal cuando manifiesta recurrir a alguien diferente de un pariente o miembro el hogar en alguna situación de urgencia.

8 La ecuación de participación será estimada para cada categoría de asociatividad. En particular, se define la variable Part cuando se incorpora la posibilidad de participación en cualquier tipo de organización; Partec, cuando se consideran las organizaciones económicas tales como: Colegio profesional, Agrupación de empresarios, Organización campesina, Grupos de adelantos rurales, Sindicatos, Cooperativa, Taller productivo, Laboral o Microempresa; Partre, en el caso de las organizaciones que buscan instancias recreativas-culturales, las cuales comprenden: Club Deportivo y recreativo, Agrupación cultural o relacionada con la creación artística, Grupo de juegos, hobby o pasatiempos; y Partve, cuando se consideran las organizaciones de vecinos, de un barrio o comuna y las juntas de vecinos. 
Con respecto a las características del hogar, se definen las variables $H O G 1_{i}$ para indicar el número de miembros del hogar entre 0-10 años, $H O G 2_{i}$ entre 11-17 años, $\mathrm{HOG}_{i}$ entre $18-24$ años, $\mathrm{HOG}_{i}$ entre $25-50$ años y $\mathrm{HOG}_{i}$ para los mayores de 50 años en el hogar. A través de estas variables se quiere evaluar si la composición de los miembros del hogar por edades genera diferencias en el costo de oportunidad al jefe de hogar (Weinberger y Jütting, 1999).

Unido a lo anterior, se tiene la variable $D E S C_{i}$, que hace referencia al número de personas desempleadas en el hogar; y la variable $L N(I N G)_{i}$, que hace referencia al logaritmo natural de los ingresos totales del hogar. Ambas variables constituyen una medida del costo de oportunidad del tiempo (Weinberger y Jütting, 1999; Lederman, 2005).

El capital social puede depreciarse como consecuencia de un desgaste en las redes sociales, situación que tiene lugar en presencia de alta movilidad de los residentes de una comunidad. Para medir la presencia de depreciación se incluye la variable dicotómica $C A S A_{i}$ que toma el valor de 1 si el jefe del hogar habita una vivienda propia, y 0 en otro caso. Con respecto a las características del lugar de residencia, se define la variable dicotómica $R U R A L_{j}$, que toma el valor de 1 si el jefe de hogar reside en una zona rural, y 0 en una zona urbana. Las características antes señaladas se asocian a una menor probabilidad de movilidad, lo que permitiría aprovechar de mejor manera los procesos asociativos (Glaeser et al., 2002; Lederman, 2005).

Adicionalmente, se incluyen dos variables a nivel provincial: la tasa de desempleo, denotada por $D E S_{j}$; y la desigualdad en el ingreso $D E S I G_{j}$, medida a través del coeficiente de Gini, las cuales permiten evaluar el efecto de la heterogeneidad de los individuos en la participación (Lederman, 2005; Alesina y La Ferrara, 2000b).

Finalmente, se incluye un conjunto de variables dicotómicas que reflejan la región de residencia del hogar: $R E G 3_{j}$ para denotar la Región de Atacama, $R E G 7_{j}$ para denotar la Región del Maule y $R E G 8_{j}$ para representar a la Región del Bío Bío; estas variables toman el valor de 1 cuando el individuo pertenece a la región mencionada, y 0 cuando pertenece a la Región Metropolitana.

Con respecto al bienestar económico, la literatura recomienda la utilización de medidas de gasto de consumo de los hogares. Sin embargo, debido a que la Encuesta Panel CASEN no registra información de este tipo, se utilizará como proxy el ingreso total de los hogares. La ecuación a estimar es de la forma:

$$
\begin{aligned}
& L N(I N G)=f\left(E D A D_{i}, E D A D_{i}^{2}, E S C_{i}, E S C_{i}^{2}, H_{i} M_{i}, N E S T_{i},\right. \\
& H O G 1_{i}, H O G 2_{i}, H O G 3_{i}, H O 4_{i}, H O G 5_{i}, D E S C_{i}, C A S A_{i}, N P R O F_{i}, \\
& \left.I A C_{i}, \text { RURAL }_{j}, D E S_{j}, D E S I G_{j}, R E G 3, R E G 7, R E G 8, \text { Part }_{i}\right),
\end{aligned}
$$

donde: $L N(I N G)$ representa el logaritmo natural del ingreso total del hogar. Con respecto a la medida de capital humano, se define la variable $N P R O F_{i}$, que hace referencia al número de profesionales en el hogar ${ }^{9}$. En relación al capital físico, se construyó un índice de activos $\left(I A C_{i}\right)$ a partir del análisis de componentes

9 Corresponde al número de miembros del hogar que completaron estudios superiores. 
principales; para ello se utilizó la información disponible con respecto a la propiedad de activos durables y a las condiciones de habitabilidad de la vivienda (Filmer y Pritchett, 2001). Finalmente, con el propósito de evaluar el efecto de la participación sobre el bienestar considerando cada categoría de asociatividad, se definen las variables Part, Partec, Partre y Partve, las cuales hacen referencia a la participación en cualquier tipo de organización, en organizaciones económicas, recreativo-culturales y vecinales, respectivamente.

\section{Análisis y Discusión de Resultados}

Con el objetivo de determinar si se tiene un modelo de participación y un modelo de ingreso, o un modelo simultáneo para el total de las organizaciones y para cada tipo de organización, se aplicaron test de sobreidentificación para validar la utilización de los instrumentos seleccionados y test de exogeneidad para evaluar la existencia de una relación simultánea entre el ingreso y la participación.

Con respecto al set de regresores exógenos, se consideraron las variables categóricas $S E G_{i}$ y $V E C_{i}$ y las variables dicotómicas $S A L U D_{i}$ y $A P O Y O_{i}$ en la instrumentación de la variable participación, y las variables NPROF e IAC en la instrumentación del ingreso. Para evaluar la pertinencia de las variables elegidas para instrumentar las variables endógenas sospechosas (participación e ingreso) se aplicó un test de sobreidentificación para una regresión estimada vía variables instrumentales, siguiendo la versión desarrollada en Sargan (1958) y Basmann (1960) para el caso del ingreso, y las versiones para modelos de ecuaciones simultáneas con variable dependiente cualitativa de Newey (1987) y Lee (1992) para el caso de la participación. De acuerdo con ambos test, si no es posible rechazar la hipótesis nula se tiene evidencia para aprobar la validez de los instrumentos utilizados. Los resultados de estos test son presentados en la Tabla 1.

TABLA 1

TEST DE SOBRE IDENTIFICACIÓN DE BASMANN (1960) Y LEE (1992)

\begin{tabular}{|c|c|c|c|c|c|c|c|c|}
\hline \multirow{2}{*}{ Estadístico } & \multicolumn{2}{|c|}{$\begin{array}{c}\text { Todas las } \\
\text { Organizaciones }\end{array}$} & \multicolumn{2}{|c|}{$\begin{array}{l}\text { Organizaciones } \\
\text { Económicas }\end{array}$} & \multicolumn{2}{|c|}{$\begin{array}{l}\text { Organizaciones } \\
\text { Vecinales }\end{array}$} & \multicolumn{2}{|c|}{$\begin{array}{l}\text { Organizaciones } \\
\text { Recreativas }\end{array}$} \\
\hline & Basmann & Lee & Basmann & Lee & Basmann & Lee & Basmann & Lee \\
\hline Chi-cuadrado & 5,275 & 0,067 & 1,218 & 0,064 & 4,75 & 0,227 & 4,831 & 0,302 \\
\hline Valor p & 0,1527 & 0,7955 & 0,7487 & 0,8001 & 0,1910 & 0,6335 & 0,1846 & 0,5828 \\
\hline
\end{tabular}

Fuente: Elaboración propia.

Como puede observarse, tanto para el caso de la ecuación de ingreso como para la ecuación de participación no se puede rechazar la hipótesis nula con un $95 \%$ de confianza, lo que indica que las variables elegidas son instrumentos 
válidos. Una vez demostrada la validez de los instrumentos utilizados, se evaluó la existencia de endogeneidad del ingreso en la ecuación de participación, a partir del test de Smith-Blundell (1986), que es una adaptación del test de Hausman para el caso de modelos Probit o Tobit. De acuerdo con este test, si no se puede rechazar la hipótesis se concluye que la variable bajo sospecha es exógena. La Tabla 2 resume los resultados de este test.

TABLA 2

TEST DE EXOGENEIDAD DEL INGRESO DE SMITH-BLUNDELL (1986)

\begin{tabular}{|lcccc|}
\hline \multicolumn{1}{|c}{ Estadístico } & $\begin{array}{c}\text { Todas las } \\
\text { Organizaciones }\end{array}$ & $\begin{array}{c}\text { Organizaciones } \\
\text { Económicas }\end{array}$ & $\begin{array}{c}\text { Organizaciones } \\
\text { Recreativas }\end{array}$ & $\begin{array}{c}\text { Organizaciones } \\
\text { Vecinales }\end{array}$ \\
\hline Chi-cuadrado & 3,8902 & 2,8075 & 6,8241 & 0,3985 \\
Valor $\mathrm{p}$ & 0,0486 & 0,0938 & 0,009 & 0,5279 \\
\hline
\end{tabular}

Fuente: Elaboración propia.

Como puede observarse, en el caso de las organizaciones económicas y vecinales no se puede rechazar la hipótesis nula con un $95 \%$ de confianza, lo que permite concluir que el ingreso es exógeno, por lo que se tiene un modelo de participación y un modelo de ingresos en forma separada. Los resultados de estos modelos son presentados en las Tablas 3 y 4.

Por otra parte, en el caso de todas las organizaciones y de las organizaciones recreativo-culturales, se encontró evidencia a favor de la endogeneidad del ingreso en la ecuación de participación, por lo que se tiene un modelo de ecuaciones simultáneas. Para corregir los sesgos derivados de la doble causalidad entre estas variables se estimó un modelo simultáneo utilizando estimadores Two Stage Probit Least Squares, ya que permiten estimar sistemas que incluyen variables dependientes continuas y dicotómicas. Los resultados de este modelo son presentados en la Tabla $5^{10}$.

En relación a los determinantes de la participación, los resultados muestran diferencias para las distintas categorías de organizaciones. Particularmente para el caso de todas las organizaciones y de las organizaciones vecinales, se puede observar una relación no lineal entre la edad y el capital social, lo que evidencia que en las fases iniciales de la vida los individuos tienden a participar más, mientras en la edad madura se observa una disminución en la participación.

De manera análoga, en el caso de las organizaciones vecinales y recreativoculturales existe también una relación no lineal entre los años de escolaridad y el capital social, lo que evidencia que los individuos con mayor capital humano, es decir, aquellos que tienen un mejor lenguaje y mayores habilidades técnicas y comunicacionales, deciden participar más en las organizaciones que involucran interacciones sociales. En el caso de la asociatividad económica, se tiene el

10 Es posible que las estimaciones presenten problemas de sesgo o de eficiencia debido a que los modelos combinan un conjunto de variables continuas con variables dicotómicas. 
TABLA 3

DETERMINANTES DE LA PARTICIPACIÓN POR TIPO DE ORGANIZACIÓN (PROBIT)

\begin{tabular}{|c|c|c|}
\hline Variables Explicativas & $\begin{array}{l}\text { Organizaciones } \\
\text { Económicas }\end{array}$ & $\begin{array}{c}\text { Organizaciones } \\
\text { Vecinales }\end{array}$ \\
\hline CONST & $-5,8361 * *$ & $-4,0073 * *$ \\
\hline \multicolumn{3}{|c|}{ Características Individuales } \\
\hline EDAD & $-0,0034$ & $0,0526 * *$ \\
\hline EDAD2 & $-0,00000215$ & $-0,0004 * *$ \\
\hline $\mathrm{ESC}$ & $-0,0735+$ & $0,0516^{*}$ \\
\hline ESC2 & $0,0062 * *$ & $-0,0032 *$ \\
\hline HOM & $-0,0812$ & 0,0582 \\
\hline SEG & $-0,0595$ & $0,1487 * *$ \\
\hline VEC & $-0,0879$ & $0,1359 * *$ \\
\hline SALUD & 0,1348 & $-0,1102+$ \\
\hline APOYO & $0,2346^{*}$ & $0,1526 * *$ \\
\hline NEST & $0,4424 * *$ & $-0,0283$ \\
\hline \multicolumn{3}{|c|}{ Características Familiares } \\
\hline HOG1 & $-0,1099$ & 0,0320 \\
\hline HOG2 & 0,0284 & $-0,0251$ \\
\hline HOG3 & $-0,0594$ & $0,0845^{*}$ \\
\hline HOG4 & $-0,0276$ & $-0,0146$ \\
\hline HOG5 & 0,0044 & 0,0417 \\
\hline DESC & $0,2205+$ & $-0,1060$ \\
\hline CASA & 0,0673 & $0,3885 * *$ \\
\hline LN(ING) & $0,2619 * *$ & $-0,0467$ \\
\hline \multicolumn{3}{|c|}{ Características Comunidad } \\
\hline RURAL & 0,1014 & $0,6695 * *$ \\
\hline DES & $-0,0946 * *$ & 0,0237 \\
\hline DESIG & 0,0349 & 0,8029 \\
\hline REG3 & $0,4116+$ & $0,5876 * *$ \\
\hline REG7 & $0,3335+$ & $0,3931 * *$ \\
\hline REG8 & $0,9348 * *$ & $0,5464 * *$ \\
\hline Observaciones & 3.766 & 3.766 \\
\hline Pseudo $\mathrm{R}^{2}$ & 0,1806 & 0,1270 \\
\hline
\end{tabular}

Fuente: Elaboración propia.

Notas:

** Estadísticamente significativos al 99\% de confianza; *Estadísticamente significativos al 95\% de confianza; ${ }^{+}$Estadísticamente significativos al $90 \%$ de confianza. 
TABLA 4

DETERMINANTES DEL BIENESTAR MATERIAL POR TIPO DE ORGANIZACIÓN (OLS)

\begin{tabular}{|c|c|c|}
\hline Variables Explicativas & Económicas & Vecinales \\
\hline CONST & $11,9368 * *$ & $11,9053 * *$ \\
\hline \multicolumn{3}{|c|}{ Características Individuales } \\
\hline EDAD & $-0,0099$ & $-0,0093$ \\
\hline EDAD2 & 0,0001 & 0,0002 \\
\hline ESC & 0,0170 & 0,0164 \\
\hline $\mathrm{ESC} 2$ & 0,0005 & 0,0005 \\
\hline HOM & 0,0034 & 0,0032 \\
\hline NEST & $0,2337 * *$ & $0,2364 * *$ \\
\hline \multicolumn{3}{|c|}{ Características Familiares } \\
\hline HOG1 & 0,0091 & 0,0088 \\
\hline HOG2 & $-0,0089$ & $-0,0089$ \\
\hline HOG3 & $0,1485^{* *}$ & $0,1493 * *$ \\
\hline HOG4 & $0,2792 * *$ & $0,2789 * *$ \\
\hline HOG5 & $0,2522 * *$ & $0,2527 * *$ \\
\hline DESC & $-0,2985^{* *}$ & $-0,2986 * *$ \\
\hline CASA & $0,4299 * *$ & $0,4346 * *$ \\
\hline NPROF & $0,1451 * *$ & $0,1483 * *$ \\
\hline IAC & $0,2246^{* *}$ & $0,2253 * *$ \\
\hline \multicolumn{3}{|c|}{ Características Comunidad } \\
\hline RURAL & $-0,0397$ & $-0,0282$ \\
\hline DES & $-0,0036$ & $-0,0042$ \\
\hline DESIG & $-1,6377 * *$ & $-1,6130 * *$ \\
\hline REG3 & $-0,0232$ & $-0,0119$ \\
\hline REG7 & $-0,2082 * *$ & $-0,2017 * *$ \\
\hline REG8 & $-0,1275^{* *}$ & $-0,1135^{* *}$ \\
\hline \multicolumn{3}{|c|}{ Participación } \\
\hline Partec & $0,1516^{*}$ & \\
\hline Partve & & $-0,0589$ \\
\hline Observaciones & 3.766 & 3.766 \\
\hline $\mathrm{R}^{2}$ & 0,3101 & 0,3100 \\
\hline
\end{tabular}

Fuente: Elaboración propia.

Notas:

**Estadísticamente significativos al 99\% de confianza; *Estadísticamente significativos al $95 \%$ de confianza; +Estadísticamente significativos al $90 \%$ de confianza. 
TABLA 5

ESTIMACIÓN SIMULTÁNEA PARTICIPACIÓN-BIENESTAR MATERIAL

(Two Stage Probit Least Squares)

\begin{tabular}{|c|c|c|c|c|}
\hline \multirow{2}{*}{$\begin{array}{l}\text { Variables } \\
\text { Ecuación }\end{array}$} & \multicolumn{2}{|c|}{ Todas } & \multicolumn{2}{|c|}{ Recreativo-Culturales } \\
\hline & Participación & Bienestar & Participación & Bienestar \\
\hline \multicolumn{5}{|c|}{ Características Individuales } \\
\hline CONST & $-5,4596 * *$ & $11,8901 * *$ & $-7,7971 * *$ & $11,6186^{* *}$ \\
\hline EDAD & $0,0465 * *$ & $-0,0091$ & $-0,0015$ & $-0,0105$ \\
\hline EDAD2 & $-0,0003 * *$ & $0,0001+$ & $-0,00002$ & $0,0001 * *$ \\
\hline ESC & 0,0123 & 0,0162 & $0,0912 * *$ & 0,0284 \\
\hline ESC2 & $-0,0005$ & 0,0006 & $-0,0066^{* *}$ & $-0,0002$ \\
\hline HOM & $-0,0373$ & 0,0019 & $0,5903 * *$ & 0,0814 \\
\hline SEG & $0,0831 * *$ & & 0,0550 & \\
\hline VEC & $0,0654+$ & & 0,0572 & \\
\hline SALUD & 0,0320 & & 0,0728 & \\
\hline APOYO & $0,1380 * *$ & & 0,0375 & \\
\hline NEST & $-0,0337$ & $0,2369 * *$ & $-0,0708$ & $0,2410 * *$ \\
\hline \multicolumn{5}{|c|}{ Características Familiares } \\
\hline HOG1 & 0,0180 & 0,0088 & $-0,0186$ & 0,0063 \\
\hline HOG2 & 0,0168 & $-0,0084$ & $0,1088 * *$ & 0,0049 \\
\hline HOG3 & 0,0411 & $0,1495 * *$ & $-0,0302$ & $0,1531 * *$ \\
\hline HOG4 & $-0,0868+$ & $0,2787 * *$ & $-0,1580 * *$ & $0,2747 * *$ \\
\hline HOG5 & $-0,1580 * *$ & $0,2503 * *$ & $-0,3114 * *$ & $0,2256^{* *}$ \\
\hline DESC & 0,0095 & $-0,2984 * *$ & 0,0451 & $-0,3096 * *$ \\
\hline CASA & 0,0611 & $0,4331 * *$ & $-0,3842 * *$ & $0,4034 * *$ \\
\hline E_LN(ING) & $0,2118+$ & & $0,4430 * *$ & \\
\hline NPROF & & $0,1490 * *$ & & $0,1507 * *$ \\
\hline IAC & & $0,2265 * *$ & & $0,2404 * *$ \\
\hline \multicolumn{5}{|c|}{ Características Comunidad } \\
\hline RURAL & $0,4503 * *$ & $-0,0314$ & 0,0094 & $-0,0387$ \\
\hline DES & $-0,0285+$ & $-0,005$ & $-0,0307$ & $-0,0090$ \\
\hline DESIG & $1,6227+$ & $-1,6111 * *$ & 2,0331 & $-1,4632+$ \\
\hline REG3 & $0,3352 * *$ & $-0,0138$ & 0,1932 & 0,0086 \\
\hline REG7 & $0,2250 * *$ & $-0,2025 * *$ & $0,2215+$ & $-0,1860 * *$ \\
\hline REG8 & $0,5861 * *$ & $-0,1101$ & $0,2767 * *$ & $-0,0877$ \\
\hline \multicolumn{5}{|c|}{ Participación } \\
\hline E_Part & & $-0,0162$ & & \\
\hline E_Partre & & & & $-0,1338$ \\
\hline
\end{tabular}

Fuente: Elaboración propia.

Notas:

**Estadísticamente significativo al $99 \%$ de confianza; *Estadísticamente significativo al $95 \%$ de confianza; + Estadísticamente significativos al $90 \%$ de confianza. 
efecto contrario, por lo que se presume que en la medida en que se adquieren mayores niveles de educación, los individuos pueden acceder a mayores y mejores oportunidades para conseguir sus objetivos sin necesidad de entablar una acción colectiva.

Por otra parte, en el caso de todas las organizaciones y de las organizaciones vecinales se encontró que las variables relacionadas a la percepción que tienen los individuos con respecto a la seguridad en su barrio y a sus relaciones con los vecinos, ambas proxy de la confianza interpersonal, tienen un efecto positivo en la probabilidad de participar; lo anterior se debe a que individuos que habitan en entornos más seguros son más propensos a confiar en los demás y, por lo tanto, están dispuestos a compartir con mayor facilidad los riesgos asociados a la participación, particularmente cuando ésta involucra personas cercanas a su entorno. Una situación similar se tiene en el caso de la variable $A P O Y O_{i}$, también proxy de la confianza, la cual es estadísticamente significativa para el caso de todas las organizaciones, organizaciones económicas y vecinales.

Con respecto a la ocupación, se tiene evidencia de una relación positiva entre la estabilidad del empleo y la participación en organizaciones económicas y con fines recreativo-culturales. En el caso de las variables asociadas al hogar, se encontró que en familias cuyos integrantes se encuentran entre los 18-24 años de edad, se tiene una mayor probabilidad de participar en organizaciones vecinales; y si sus miembros tienen 24 años y más, se observa una menor probabilidad de participar en organizaciones recreativo-culturales y en todas las organizaciones.

Asimismo, se encontró que la presencia de miembros desempleados en el hogar tiene una relación positiva con la participación en organizaciones con fines económicos; lo anterior se debe a que esta alternativa de participación puede ser vista por los individuos como una fuente potencial para obtener ingresos, a diferencia de lo que sucede en el resto de organizaciones, las cuales imponen altos costos de oportunidad.

Por otra parte, se encontró una relación positiva entre la tenencia de una vivienda propia y la participación en organizaciones vecinales. Sin embargo, esta relación se vuelve negativa y significativa cuando las estimaciones consideran la participación en organizaciones recreativo-culturales.

Finalmente, se tiene una relación positiva entre la participación en organizaciones de índole económica y recreativo-cultural con el logaritmo del ingreso; en este sentido, las familias con mayores ingresos enfrentan menores restricciones económicas para acceder a un conjunto de bienes. De esta manera, la participación en organizaciones recreativas puede ser vista como un bien superior (Grootaert y Narayan, 2004), mientras que en el caso de las organizaciones económicas podrían existir mayores costos de acceso que limiten la participación de grupos de menores ingresos.

Con respecto a las variables asociadas a la comunidad de residencia del jefe de hogar, se encontró que los individuos que residen en zonas rurales tienen una mayor probabilidad de participar en todas las organizaciones y particularmente en las organizaciones vecinales; lo anterior se debe a que las personas que habitan estas zonas tienen mayor capacidad para generar redes sociales debido a que, en promedio, presentan un mayor tiempo de permanencia en su localidad con relación a los individuos que habitan en la zona urbana, y en promedio, enfrentan menores costos de traslado. 
Por otra parte, se evidencia que en las provincias con mayores tasas de desempleo se tiene una menor participación en organizaciones económicas, lo que viene a ratificar la estrecha relación entre oportunidades de empleo y la pertenencia a este tipo de organizaciones. No existe evidencia de significancia estadística entre la medida de desigualdad y participación. Finalmente, se encontró que los individuos de las regiones de Atacama, Maule y Bío-Bío tienden a participar más en organizaciones, en comparación con los individuos que habitan en la Región Metropolitana.

En relación a los determinantes del ingreso, específicamente en lo que respecta a las variables individuales, se evidencia que los individuos que poseen un trabajo estable exhiben mayores niveles de ingreso.

Por otra parte, las variables asociadas al hogar evidencian que existe una relación positiva entre el ingreso familiar y la edad de sus miembros: a mayor edad, se tienen mayores posibilidades de obtener un trabajo remunerado, lo que incrementa el ingreso del hogar, y por consiguiente el bienestar. Una situación similar se observa en el caso de familias cuyos miembros poseen estudios superiores (profesionales); esta medida constituye una proxy del nivel de capital humano de la familia. Asimismo, se tiene una relación inversa entre la presencia de miembros desempleados en el hogar y la posibilidad de generar ingresos.

Unido a lo anterior, se evidencia que las familias que poseen vivienda propia pueden disfrutar de una proporción mayor de su ingreso en el consumo de otros bienes, lo que tiene efectos directos e indirectos en el bienestar. Finalmente, se tiene una relación directa entre el índice de activos y el bienestar del hogar; esta medida constituye una proxy del nivel de capital físico de la familia.

Con respecto a las características de la comunidad, se evidencia que la desigualdad en el ingreso en la provincia de residencia del jefe de hogar tiene un efecto negativo en el bienestar familiar, y que las familias que habitan en la regiones del Maule y Bío-Bío poseen menores niveles de ingreso con respecto a los individuos que habitan en la Región Metropolitana. Finalmente, se encontró que la participación en organizaciones con fines económicos tiene un impacto positivo en el ingreso de las familias, lo que sugiere que la existencia y promoción de este tipo de instancias puede llevar a una mejora en el bienestar económico de los hogares y, por consiguiente, en su calidad de vida, medido a través del ingreso.

\section{Conclusiones}

La asociatividad puede ser vista como una alternativa para mejorar el bienestar económico de los hogares, al entregar a sus miembros mayores oportunidades para el acceso a recursos, a la vez que fomenta comportamientos cooperativos que fortalecen las decisiones que involucran el bienestar colectivo. Asimismo, la generación de capital social en una sociedad puede facilitar el proceso de devolución de poder a la ciudadanía, motivando de esta manera la solución descentralizada de conflictos sociales que involucran intereses contrapuestos. Conocer el proceso que describe la decisión de participar en organizaciones, así como la diferenciación de estos procesos de acuerdo a los distintos ámbitos asociativos, es relevante para el diseño de iniciativas que promocionen y fortalezcan estas instancias como una vía para la creación de vínculos de confianza, aspecto 
de gran relevancia si se quiere avanzar en los procesos de desarrollo. Especial importancia cobra el estudio del conjunto de organizaciones que promueven mayores oportunidades orientadas a fortalecer y ampliar las capacidades de las familias para generar ingresos.

Se estimaron modelos de participación e ingreso en el caso de las organizaciones económicas y vecinales, y un modelo de ecuaciones simultáneas en el caso de las organizaciones recreativo-culturales, debido a la existencia de endogeneidad del ingreso en la ecuación de participación. El modelo simultáneo propuesto incluye estimadores Two Stage Probit Least Squares, que son adecuados para corregir los sesgos derivados de la simultaneidad cuando una variable dependiente es dicotómica y la otra continua.

En relación a los determinantes de la participación, se destaca la importancia de aspectos relacionados con la seguridad y convivencia en el entorno comunitario, especialmente cuando se busca congregar acciones colectivas desde organizaciones construidas desde los barrios. Adicionalmente, la tenencia de una vivienda propia y la residencia en zonas rurales aparecen también como variables relevantes para explicar este tipo de participación. Asimismo, variables vinculadas al ámbito laboral, como es el caso de la estabilidad del empleo y el nivel de desocupación, se distinguen como determinantes de la participación en organizaciones económicas. Finalmente, la composición de los miembros del hogar, la escolaridad y el ser propietario de la vivienda destacan en la explicación de la participación en organizaciones recreativo-culturales.

Por otra parte, los resultados muestran un efecto positivo y significativo del ingreso sobre la participación, validando la tesis planteada en estudios previos en relación a que la participación puede ser tratada como un bien superior.

Con respecto a los efectos de la participación en el bienestar, se encuentra que el capital social, medido a través de la participación en organizaciones, no tiene una relación con el ingreso cuando no se hace distinción del tipo de organización. Asimismo, en el caso de las organizaciones vecinales y recreativo-culturales, no se encontró una relación significativa entre ingreso y afiliación. En el caso de las organizaciones vecinales, los individuos buscan mejoras en el entorno comunitario, mientras que en el caso de las organizaciones recreativo-culturales los individuos persiguen el acceso a nuevas oportunidades de esparcimiento, recreación y expresión artística, objetivos que no pueden ser medidos a través del ingreso, proxy del bienestar económico en el presente estudio.

Pese a lo anterior, en el caso de las organizaciones económicas los resultados evidencian una relación significativa entre ingreso y afiliación. La pertenencia a este tipo de organizaciones podría ayudar a sus miembros a ampliar las oportunidades para la generación de ingresos. Sin embargo, dado que la participación en organizaciones de este tipo se encuentra limitada por los niveles de ingreso, se tienen restricciones para que las familias más pobres puedan tener acceso a aquellas instancias donde puedan obtener retornos económicos más altos. Este aspecto revela un desafío para orientar acciones que favorezcan el acceso de los grupos más vulnerables a organizaciones que buscan potenciar económicamente a sus miembros. 


\section{REFERENCIAS}

Agarwal, B. (2001). Participatory exclusions, community forestry, and gender: an analysis for South Asia and a conceptual framework. World Development 29 (10): 1623-1648.

Aker, J. (2007). Social networks and household welfare in Tanzania: working together to get out of poverty. Journal of African Economies (revise and resubmit).

Alesina, A. y La Ferrara, E. (2000a). Determinants of trust. Working Paper 7621, National Bureau of Economic Research.

Alesina, A. y La Ferrara, E. (2000b). Participation in heterogeneous communities, Quarterly Journal of Economics, 115 (3): 847-904.

Amemiya, T. (1979). The estimation of a simultaneous equation generalized tobit model. Journal of Econometrics 8 (1): 13-21.

Amudavi, D. (2007). The effects of farmer community group participation on rural livelihoods in Kenya. Working Paper. Department of Agricultural Education and Education, Egerton University, Kenya.

Basmann, R. (1960). On finite sample distributions of generalized classical linear identifiability test statistics. Journal of the American Statistical Association 55 (292): 650-59.

Baum, C.; Schaffer, M.; Stillman, S. y Wiggins, V. (2006). Overid: Stata module to calculate tests of overidentifying restrictions after ivreg, ivreg2, ivprobit, ivtobit, reg3. http://ideas.repec.org/c/boc/bocode/s396802.html.

Bebbington, A. (2005). Estrategias de vida y estrategias de intervención: el capital social y los programas de superación de la pobreza. Revista de la CEPAL 86: 21-46.

Behera, B. (2005). Who forms local institutions? Levels of household participation in India's Joint Forest Management program. Working Paper. Center for Development Research, University of Bonn, Germany.

Behera, B. y Engel, S. (2006). How participatory are Joint Forest Management institutions in Andhra Pradesh, India? ZEF-Discussion Papers on Development Policy 103.

Cárdenas, J. (2003). "En vos confío": An experimental exploration of the micro-foundations of trust, reciprocity and social distance in Colombia. Working Paper. CEDE, Universidad de los Andes.

Davis, K. y Negash, M. (2007). Gender, health and participation in community groups in Meru Central District, Kenia. Collective action and property right (CAPRi). Working Paper 65.

Degli, A. (2007). Do social relations affect economic welfare? A microeconomic empirical analysis, Nota di Lavoro FEEM 32, Fondazione Eni Enrico Mattei, Milano.

Filmer, D. y Pritchett, L. (2001). Estimating wealth effects without expenditure data-or tear: An application to educational enrollments in states of India. Demography, 38: 115-132.

Glaeser, E.; Laibson, D. y Sacerdote, B. (2002). An economic approach to social capital, The Economic Journal 112 (483).

Gordon, S. (2005). Confianza, capital social y desempeño de organizaciones. Revista Mexicana de Ciencias Políticas y Sociales, vol. XLVII, 193: 41-55. 
Greene, W. (1999). Análisis Econométrico. Pearson Eduación, tercera edición.

Grootaert, C. (1997). Social capital: the missing link? (In expanding the measure of wealth: indicators of environmentally sustainable development, pp. 7794). Environmentally Sustainable Development Monographs Series, $\mathrm{N}^{\mathrm{o}}$ 17. Washington DC: The World Bank.

Grootaert, C. (1999). Social capital, household welfare and poverty in Indonesia. Local Level Institutions Study. Working Paper $\mathrm{N}^{\mathrm{o}}$ 6. Washington, D.C.: The World Bank, Social Development Family, Environmentally and Socially Sustainable Development Network.

Grootaert, C.; Oh, G. y Swamy, A. (2002). Social capital, household welfare and poverty in Burkina Faso. Journal of African Economies, 2 (1): 4-38.

Grootaert, C. y Narayan, D. (2004). Local institutions, poverty and household welfare in Bolivia, World Development 32 (7): 1179-1198.

Haddad, L. y Maluccio, J. (2003). Trust, membership in groups, and household welfare: evidence from KwaZulu-Natal, South Africa. Economic Development and Cultural Change, 51 (3): 573-601.

Hardin, R. (1968). The tragedy of the commons, Science 162: 1243-1248.

$\mathrm{Hu}$, Chia-Hsin y Jones, B. (2004). An investigation into the relationship between household welfare and social capital in eastern Uganda. Final Report for SAGA Competitive Research Grants Program.

Jütting, J. (2003). Health insurances for the poor? Determinants of participation in community-based health insurances schemes in rural Senegal. Technical Paper $\mathrm{N}^{\mathrm{o}}$ 204, OECD Development Center.

Katungi, E.; Machethe, C. y Smale, M. (2007). Determinants of social capital formation in rural Uganda: Implications for group-based agricultural extension approaches. African Journal of Agricultural and Resource Economics 1 (2): 167-190.

Keshk, O. M.G. (2003). CDSIMEQ: A program to implement two-stage probit least squares, Stata Journal 3 (2): 157-167.

Khanal, B. (2005). Community forestry program in the hills of Nepal: determinants of user participation in forest management activities. Department of International Environment and Development Studies (Noragric), Norwegian University of Life Sciences (UMB).

Kliksberg, B. (1999). Capital social y cultura, claves esenciales del desarrollo. Revista de la CEPAL 69: 85-102.

Knack, S. y Keefer, P. (1997). Does social capital have an economic pay-off? A cross-country investigation. Quarterly Journal of Economics 112 (4): 1251-1288.

Kothari, U. (2001). Power knowledge and social control in participatory development. In B. Cooke y U. Kothari (eds.), Participation: the new tyranny?, pp. 139-152, Zed Books, London.

Lederman, D. (2005). Income, wealth, and socialization in Argentina. Cuadernos de Economía, 42: 3-30.

Lee, L. (1992). Amemiya's generalized least squares and tests of overidenfication in simultaneous equation models with qualitative or limited dependent variables. Econometric Reviews 11 (3): 319-328.

Maddala, G. (1983). Limited-Dependent and Qualitative Variables in Econometrics. Cambridge, M.A.: Cambridge University Press. 
Narayan, D. (1997). Voices of the poor: poverty and social capital in Tanzania. Environmentally Sustainable Studies and Monograph Series 20. The World Bank, Washington, D.C.

Narayan, D. y Pritchett, L. (1999). Cents and sociability: Household Income and Social Capital in Rural Tanzania. Economic Development and Cultural Change, 47 (4): 871-897.

Newey, W. (1987). Efficient estimation of limited dependent variable models with endogeneous explanatory variables. Journal of Econometrics 36: 231-250.

Olson, M. (1965). The logic of collective action. Cambridge: Harvard University Press.

PNUD (Programa de las Naciones Unidas para el Desarrollo (PNUD) (2000). Asociatividad y capital social. Desarrollo Humano en Chile, pp. 107172.

Portes, A. y Landolt, P. (2000). Social capital: promise and pitfalls of its role in development. Journal of Latin American Studies 32: 529-547.

Putnam, R. (1995). Bowling alone: American's declining social capital. Journal of Democracy, 6: 65-78.

Sargan, J. (1958). The estimation of economic relationships using instrumental variables. Econometrica 26: 393-415.

Smith, R. y Blundell, R. (1986). An exogeneity test for a simultaneous equation tobit model with an application to labor supply, Econometrica 54: 679685.

Townsend, R. (1994). Risk and insurance in Village India, Econometrica 62 (3): 539-591.

Weinberger, K. y Jütting, J. (1999). Determinants of participation in community based organizations: experiences from group based projects in Kashmir and Chad. International Journal of Economic Development, 1 (4).

Woolcock, M. (1998). Social capital and economic development: toward a theoretical synthesis and policy framework. Theory and Society 27: 151-208.

Yusuf, S. (2008). Social capital and social welfare in Kwara state, Nigeria. Journal of Human Economics 23 (3): 219-229. 


\section{APÉNDICE A}

\section{Two Stage Probit Least SQuares}

Dado el sistema de ecuaciones:

$$
\begin{aligned}
& y_{1 i}^{*}=\gamma_{1} y_{2 i}^{*}+\beta_{1}^{\prime} X_{1}+\varepsilon_{1} \\
& y_{2 i}^{*}=\gamma_{2} y_{1 i}^{*}+\beta_{2}^{\prime} X_{2}+\varepsilon_{2},
\end{aligned}
$$

donde: $y_{1 i}^{*}$ y $y_{2 i}^{*}$ hacen referencia a las variables endógenas del sistema. $X_{1} \mathrm{y}$ $X_{2}$ son matrices de variables exógenas; $\gamma_{1}$ y $\gamma_{2}$ son los parámetros estimados asociados a las variables endógenas; $\beta_{1}^{\prime}$ y $\beta_{2}^{\prime}$ son vectores de parámetros asociados al conjunto de variables exógenas. Finalmente, $\varepsilon_{1}$ y $\varepsilon_{2}$ corresponden al término error.

La estrategia de estimación para modelos de ecuaciones simultáneas depende de la naturaleza del problema a estimar, es decir, de la manera en que $y_{1}^{*}$ e $y_{2}^{*}$ son observados. Si ambas variables son continuas, se tiene un procedimiento estándar para modelos de ecuaciones simultáneas. Si alguna de las variables dependientes del sistema presenta una naturaleza distinta, se debe incorporar otra estrategia de estimación. En el caso en que la naturaleza de una de las variables endógenas sea dicotómica y la otra continua, el procedimiento Two Stage Probit Least Squares entrega estimadores consistentes y eficientes (Maddala, 1983). Este método adapta los procedimientos usuales de estimación de modelos de ecuaciones simultáneas, la diferencia se encuentra en el cálculo de los errores estándar.

El sistema definido por las ecuaciones (A1) y (A2), cuando el modelo considera una variable dependiente dicotómica y la otra continua, es de la forma:

$$
\begin{gathered}
y_{1 i}^{*}=\gamma_{1} y_{2 i}+\beta_{1}^{\prime} X_{1}+\varepsilon_{1} \\
y_{2 i}=\gamma_{2} y_{1 i}^{*}+\beta_{2}^{\prime} X_{2}+\varepsilon_{2},
\end{gathered}
$$

donde: $y_{2 i}$ corresponde a la variable endógena continua e $y_{1 i}^{*}$ define la variable dicotómica, la cual es observada como $y_{1 i}=1$ si $y_{1 i}^{*}>0$, y cero en otro caso. Las ecuaciones de la forma reducida son expresadas como sigue:

$$
\begin{aligned}
& y_{1 i}^{*}=\Pi_{1}^{\prime} X+v_{1} \\
& y_{2 i}=\Pi_{2}^{\prime} X+v_{2},
\end{aligned}
$$

donde: $\mathrm{X}$ corresponde a una matriz que incluye todas las variables exógenas del sistema; $\Pi_{1}^{\prime}$ у $\Pi_{2}^{\prime}$ son vectores de parámetros a ser estimados; $v_{1}$ y $v_{2}$ hacen referencia a los términos de error. Debido a que $y_{1 i}^{*}$ es observado como una variable dicotómica, el coeficiente estimado se encuentra afectado por el 
parámetro de escala, por lo que se observa $\frac{\Pi_{1}^{\prime}}{\sigma_{1}}$, donde $\sigma_{1}^{2}=\operatorname{Var}\left(v_{1}\right)$. De esta manera, la ecuación (A5) puede reescribirse como:

$$
y_{1 i}^{* *}=\frac{y_{1 i}^{*}}{\sigma_{1}}=\frac{\Pi_{1}^{\prime}}{\sigma_{1}} X+\frac{v_{1}}{\sigma_{1}}=\Pi_{1}^{\prime *} X+v_{1}^{*}
$$

Finalmente, las ecuaciones estructurales del modelo son de la forma:

$$
\begin{aligned}
& y_{1 i}^{* *}=\frac{\gamma_{1}}{\sigma_{1}} y_{2 i}+\frac{\beta_{1}^{\prime}}{\sigma_{1}} X_{1}+\frac{\varepsilon_{1}}{\sigma_{1}} \\
& y_{2 i}=\gamma_{2} \sigma_{1} y_{1 i}^{* *}+\beta_{2}^{\prime} X_{2}+\varepsilon_{2}
\end{aligned}
$$

Posteriormente se sigue un proceso de estimación en dos etapas. En una primera etapa, la ecuación (A6) es estimada por MCO y la ecuación (A7) es estimada utilizando un modelo Probit, obteniendo predicciones de cada variable endógena de la forma:

$$
\begin{gathered}
\hat{y}_{1 i}^{* *}=\hat{\Pi}_{1}^{\prime *} X \\
\hat{y}_{2 i}=\hat{\Pi}_{2}^{\prime} X
\end{gathered}
$$

Así, las variables originales definidas en (A8) y (A9) son reemplazadas por sus valores predichos, resultando lo siguiente:

$$
\begin{aligned}
& y_{1 i}^{* *}=\frac{\gamma_{1}}{\sigma_{1}} \hat{y}_{2 i}+\frac{\beta_{1}^{\prime}}{\sigma_{1}} X_{1}+\frac{\varepsilon_{1}}{\sigma_{1}}, \\
& y_{2 i}=\gamma_{2} \sigma_{1} \hat{y}_{1 i}^{* *}+\beta_{2}^{\prime} X_{2}+\varepsilon_{2}
\end{aligned}
$$

Nuevamente la ecuación (A12) es estimada a través de un modelo Probit y la ecuación (A13) por MCO. Note que los errores estándar estimados desde las ecuaciones (A12) y (A13) deben ser corregidos. Para su corrección se deriva la matriz de covarianzas asintótica siguiendo el estimador NelsonOlsen sugerido en Amemiya (1979). Así, dados los parámetros estimados del modelo, se define: 


$$
\begin{gathered}
\alpha_{1}^{\prime}=\left(\frac{\gamma_{1}}{\sigma_{1}}, \frac{\beta_{1}^{\prime}}{\sigma_{1}}\right) \\
\alpha_{2}^{\prime}=\left(\gamma_{2} \sigma_{1}, \beta_{2}^{\prime}\right) \\
c=\sigma_{2}^{2}-2 \gamma_{2} \sigma_{12} \\
d=\left(\frac{\gamma_{1}}{\sigma_{1}}\right) \sigma_{2}-2\left(\frac{\gamma_{1}}{\sigma_{1}}\right)\left(\frac{\sigma_{12}}{\sigma_{1}}\right) \\
H=\left(\Pi_{1}, J_{2}\right) \\
G=\left(\Pi_{2}, J_{1}\right) \\
V_{0}=\operatorname{Var}\left(\hat{\Pi}_{1}\right),
\end{gathered}
$$

donde: $J_{1}$ y $J_{2}$ denotan matrices tal que $X J_{1}=X_{1}$ y $X J_{2}=X_{2}, \sigma_{2}^{2}=\operatorname{Var}\left(v_{2}\right)$, $\sigma_{12}=\operatorname{Cov}\left(v_{1}, v_{2}\right)$. Finalmente, la matriz de covarianzas asintótica de $\alpha_{1}$ y $\alpha_{2}$ se expresa como:

(A14) $V\left(\hat{\alpha}_{1}\right)=\left(G^{\prime} V_{0}^{-1} G\right)^{-1}+d\left(G^{\prime} V_{0}^{-1} G\right)^{-1} G^{\prime} V_{0}^{-1}\left(X^{\prime} X\right)^{-1} V_{0}^{-1} G\left(G^{\prime} V_{0}^{-1} G\right)^{-1}$

(A15) $V\left(\hat{\alpha}_{2}\right)=c\left(H^{\prime} X^{\prime} X H\right)^{-1}+\left(\gamma_{2} \sigma_{1}\right)^{2}\left(H^{\prime} X^{\prime} X H\right)^{-1} H^{\prime} X^{\prime} V_{0} X^{\prime} X H\left(H^{\prime} X^{\prime} X H\right)^{-1}$ 
